\title{
Propylthiouracil induced lupus erythematosus in a patient with Grave's disease: a case report.
}

\author{
Authors: D.Keri,V. Hoxha, M. Kermaj, A.Zeqja, G. Husi, A. Ylli. \\ Departement of Endocrinology "Mother Teresa" Tirane, Albania
}

Introduction: Drug-induced Lupus Erythematosus (DILE) is a variant of Systemic Lupus Erythematosus that resolves within days to months after withdrawal of the drug, in a patient with no previous history of SLE.

Case report: A 60 year old female presented at Mother Theresa Hospital with toxic multinodular goiter. She received treatment with propylthiouracil and atenolol for a month, and she developed symptoms like malaise, muscle pain, shortness of breath, cough, nausea, vomiting and gastric pain. The laboratory tests revealed elevated thyroid function, elevated transaminases and pancytopenia. Abdominal ultrasound was normal. Bone marrow aspiration resulted normal. Positive direct and indirect Coombs tests confirmed the immune-mediated cause of pancytopenia, and suspicions were raised towards a drug-induced mechanism. Under these circumstances PTU was discontinued and the patient was started on prednisolone.

The next day the patient complained of left hip pain, breath shortness and the appearance of mouth ulcers. Thoraco-abdominal CT scan revealed pleural effusion, splenomegaly and coxarthritis sinister. Antinuclear antibodies resulted positive $(+++), C_{3}=121$ (79-152), $C_{4}=14,7$ (16-38). Prednisolone dose was raised. A week after, her condition improved and the blood tests started to normalize. Methimazole was started and the patient observed for adverse effects. After a year she continued on methimazole and her serologic markers resulted within ranges.

Discussion: DILE can arise months to years after exposure to drugs prescribed to treat various medical conditions. Literature describes cases where antithyroid drugs induce autoimmune disorders. The recognition of this side effect of antithyroids is important for the early interruption of the drug, which would lead to an improvemet of the situation, and avoid unnecessary tests and treatments. 